\title{
ARTICLE OPEN Voltage-driven charge-mediated fast 180 degree magnetization switching in nanoheterostructure at room temperature
}

\author{
Min Yi $\mathbb{B D}^{1}$, Hongbin Zhang ${ }^{1}$ and Bai-Xiang $\mathrm{Xu}^{1}$
}

Voltage-driven $180^{\circ}$ magnetization switching without electric current provides the possibility for revolutionizing the spintronics. We demonstrated the voltage-driven charge-mediated $180^{\circ}$ magnetization switching at room temperature by combining firstprinciples calculations and temperature-dependent magnetization dynamics simulation. The electric field $(E)$-induced interface charge is found to allow a giant modulation of the magnetic anisotropy $(K)$ of the nanomagnet. Particularly $K$ is revealed to vary linearly with respect to $E$ and the epitaxial strain. Magnetization dynamics simulations using the so-obtained $K$ show that both inplane and perpendicular $180^{\circ}$ switching can be achieved by $E$ pulses. The temperature effect renders the $180^{\circ}$ switching as probability events. Statistical analysis indicates a fast (around $4 \mathrm{~ns}$ ) and low-error-probability $180^{\circ}$ switching achievable at room temperature by controlling the magnitude of $E$ and the pulse width. The study inspires the rational design of miniaturized nanoscale spintronic devices where thermal fluctuation has a great impact.

npj Computational Materials (2017)3:38; doi:10.1038/s41524-017-0043-x

\section{INTRODUCTION}

Recently, the voltage control of magnetism without electric current has been extensively studied in order to achieve minimum power consumption and device miniaturization in next-generation of spintronics. ${ }^{1-10}$ Such a control is usually implemented through the magnetoelectric (ME) coupling in heterostructures which possess coupled magnetic and electric properties. Generally, in ME heterostructures voltage can control the magnetism through the interfacial mechanisms such as elastic coupling via strain transfer, ${ }^{11-32}$ charge modulation, ${ }^{31-43}$ interface bonding, ${ }^{44-48}$ and exchange coupling. ${ }^{29,49-52}$ For the ferromagnetic/ferroelectric heterostructures, elastic coupling mechanism is most extensively investigated, i.e., a strain generated in a ferroelectric layer by a voltage is transferred to the ferromagnetic layer through the interface and thus can be used to tailor magnetization through the magnetoelastic coupling. The elastic coupling mechanism can work at the bulk level. All the other three mechanisms are localized to the region near the interface. Among them, the exchange coupling is localized, but can generate exchange spring effects to affect the magnetization in the bulk. ${ }^{53}$

By using these various mechanisms, a voltage induced $180^{\circ}$ magnetization switching is of great interests. For example, in order to achieve a high signal-to-noise ratio in magnetic tunnel junction (MTJ), a significantly large electric resistance change of MTJ is required, which can only be achieved by a $180^{\circ}$ switching in the free layer of MTJ. Trailblazing experiments have demonstrated strain-mediated and exchange-coupling-mediated voltage-driven/ assisted magnetization switching in $\mathrm{Ni} / \mathrm{BaTiO}_{3}{ }^{23} \mathrm{Co} / \mathrm{PMN}-\mathrm{PT}{ }^{43}$ and $\mathrm{CoFe} / \mathrm{BiFeO}_{3}{ }^{50,51}$ heterostructures, respectively. Nevertheless, the voltage-driven full $180^{\circ}$ switching is not achieved in some experiments. For example, in the $\mathrm{CoFe} / \mathrm{BiFeO}_{3}$ only a net magnetization reversal is realized. ${ }^{50}$ The electric field is found to decrease the coercivity, but the switching is still achieved by magnetic field. ${ }^{40,51}$ Also some forms of magnetic fields are involved in experiments. ${ }^{23,43,50}$ From the theoretical point, a large number of studies are devoted to the strain-mediated $180^{\circ}$ switching either by designing the shape of magnets ${ }^{30}$ or by using the 3D precessional switching dynamics. ${ }^{13-15,18,19,24,25,27}$

Alternatively, interface charge modulation has been deemed as an ideal way for realizing magnetic switching in thin film heterostructures. ${ }^{33-43}$ But plenty of experimental and theoretical investigations show charge-modulated coercivity or chargemediated magnetization switching between two states which are not exactly rotated by $180^{\circ} .33,34,37,39-41$ For example, an electric field induced change in the number of unpaired $d$ electrons in FePt and FePd can induce their coercivity change by several percent, but the voltage-driven magnetization switching is not addressed. ${ }^{33}$ Controlling the charge carrier concentration in $(\mathrm{Ga}$, $\mathrm{Mn})$ As by an electric field can manipulate the magnetization direction by only $10^{\circ} .{ }^{39}$ In Fe/MgO system, through the voltage control of relative occupation of $3 d$ orbitals in interfacial Fe atoms, an electric field of $0.1 \mathrm{~V} / \mathrm{nm}$ is shown to cause a $40 \%$ change in the magnetic anisotropy of $\mathrm{Fe}^{37} \mathrm{In} \mathrm{CoFeB} / \mathrm{MgO} / \mathrm{CoFeB}$, electric field is shown to tune the coercivity for magnetization switching driven by a magnetic field. ${ }^{40}$ Coherent control of magnetization using voltage signals is realized in $\mathrm{FeCo} / \mathrm{MgO}$ with an additional magnetic field of $70 \mathrm{mT}$ and switching angle less than $180^{\circ} .{ }^{41}$ Meanwhile, most studies deal with the thin film structure with a lateral size of several hundred nanometers to several micrometers or with a thickness of several tens nanometers. In such cases, the magnet volume is relatively large so that the temperature effect on the switching dynamics is often ignored, $11,13-15,18-20,25,30,34,52$ or shown to be not so strong. ${ }^{24,27}$ However, in terms of the device miniaturization, if spintronic devices are further scaled down to nanodot shape, i.e., with the size of several tens of nanometers in the lateral direction, the huge reduction of the magnet volume will intensify the effects of temperature-induced thermal fluctuations. For instance, the granular film with $L 1_{0}$-ordered $\mathrm{FePt}$

${ }^{1}$ Department of Materials and Earth Sciences, Technische Universität Darmstadt, 64287 Darmstadt, Germany

Correspondence: Min Yi (yi@mfm.tu-darmstadt.de)

Received: 17 May 2017 Revised: 25 August 2017 Accepted: 28 August 2017

Published online: 22 September 2017 
epitaxially grown on $\mathrm{MgO}$ substrate, which has been considered as a promising candidate for memory devices, usually contains FePt nanograins with a size of several nanometers to several tens of nanometers. If such a small-scaled $\mathrm{FePt} / \mathrm{MgO}$ heterostructure is utilized, considering the effect of thermal fluctuations on the magnetization dynamics is indispensable.

In this work, we take epitaxial Pt/FePt/MgO heterostructures as a model system with a lateral size of several tens of nanometers. An elliptical shape of FePt (Fig. 1b) is chosen, which allows the magnetic anisotropy within the $x-y$ plane and thus the in-plane magnetization switching. The electric field induced change in the $\mathrm{FePt} / \mathrm{MgO}$ interface charge can tailor the magnetocrystalline anisotropy energy (MAE) of FePt layer, thus manipulating the magnetic easy axis of the heterostructure system. Meanwhile, the epitaxial strain plays a role in determining whether the equilibrium magnetic state without electric field is in-plane or perpendicular. By controlling the electric field, epitaxial strain, and magnetization dynamics, the in-plane and perpendicular $180^{\circ}$ magnetization switching (Fig. 1c) is achievable in the case of in-plane and perpendicular equilibrium magnetic state, respectively. Specifically, combining first-principles calculation and temperature-dependent magnetization dynamics, we demonstrate the in-plane and perpendicular $180^{\circ}$ magnetization switching at room temperature. It is anticipated that the present study provides valuable insight into the design of voltage control of both in-plane and perpendicular $180^{\circ}$ switching at room temperature for achieving low-power, high-speed, non-volatile, and highly compact spintronics.

\section{RESULTS}

The material model system for first-principles calculations is shown in Fig. 1a. Since the lattice parameter of bulk $\mathrm{MgO}$ is $\sim 4.22$ $\AA$ and that of FePt is $\sim 3.86 \AA$, the lattice mismatch is up to $\sim 8.5 \%$. In experiments, $\mathrm{MgO}$ with tunable lattice strain can be epitaxially grown at different temperature on different substrates like $\mathrm{Cu}^{54}$ $\mathrm{Ge}^{55} \mathrm{Si}^{56}$ etc. So different in-plane epitaxial strains $\left(\varepsilon_{\mathrm{MgO}}\right)$ relative to the equilibrium lattice parameter in $\mathrm{MgO}$ at different FePt layer number $(n)$ are adopted to study their effects. The material system for magnetization dynamics analysis is shown in Fig. 1b. The FePt nanomagnet is an elliptical cylinder with height $t(n)$, semimajor axis $a$, and semiminor axis $b$. Two angles are used to describe the magnetization state, as presented in the Methods section.

Figure 2 presents the first-principles results when $\varepsilon_{\mathrm{MgO}}=-3.26 \%$ and $n=5$. From the electrostatic potential distribution obtained from first-principles calculation in Fig. 2a, we can estimate the external electric field as $0.9 \mathrm{~V} / \mathrm{nm}$. Under this electric field, the charge density around $\mathrm{Pt}$ and Fe atoms which are close to the interface is evidently decreased. The effect of electric field extends to the first two layers of FePt next to the interface. The charge change will affect the $d$-orbital hybridization and thus the MAE, as shown the orbital-resolved MAE in Fig. S1 (in Supplementary Information, $\mathrm{SI}$ ). The atom-resolved MAE analysis in Fig. $2 \mathrm{~d}$ indicates the dominative role of the interfacial Pt (Pt1) in the perpendicular magnetic anisotropy. The electric field induced change in MAE of Pt1 controls the MAE change of the system. Orbital-resolved MAE analysis in Fig. S1 (in SI) further confirms the largest MAE contribution of the hybridization between $d_{y z}$ and $d_{z^{2}}$ of Pt1. MAE of the system is shown to be linearly dependent on the strength of the electric fields, as presented in Fig. 2c. A giant modulation of MAE by applying electric fields is observed, with a amplitude as high as $322 \mathrm{fJ} / \mathrm{Vm}$.

We then calculate saturation magnetization $\left(M_{s}\right.$, total magnetic moment divided by FePt volume) and $K$ (MAE divided by FePt volume) under different $\varepsilon_{\mathrm{MgO}}, E$ and $n$, as presented in Fig. S2 (in SI) and Fig. 3, respectively. $M_{s}$ is found to be not significantly affected by $E$, but depend more on $\varepsilon_{\mathrm{MgO}}$ and $n$ (Fig. S2 in SI). It can be seen from Fig. 3 that $K$ varies linearly with respect to both $\varepsilon_{\mathrm{MgO}}$ and $n$, as indicated by the fitted linear functions therein. $K$ is more sensitive to $\varepsilon_{\mathrm{MgO}}$ and can be altered from approximately 10 to -5 $\mathrm{MJ} / \mathrm{m}^{3}$ when $\varepsilon_{\mathrm{MgO}}$ is increased from -7 to $-1 \%$. The strong dependence of $K$ on $\varepsilon_{\mathrm{MgO}}$ intrigues extensive studies of the strainmediated magnetization switching. Positive electric field results in an increment in $K$. The charge-mediated dependence of $K$ on $E$ provides the feasibility to switch the magnetization. For example, using the model in Fig. $1 \mathrm{~b}$, we find that through the chargemediated $K$ change, the electric field can be used to tailor the magnetic hysteresis (Fig. S3 in SI).

From the perspective of practical applications of magnetic states in data storage, the energy barrier is usually required to exceed $40 k_{\mathrm{B}} T$ to make an initial equilibrium magnetization sufficiently stable. For this requirement, in the case of no electric field $(E=0)$, we calculate the energy barrier $(\Delta G)$ of initial magnetization stabilized in both in-plane $x$ and perpendicular $z$ directions, in order to check the thermal stability of the initial magnetization. As for the initial equilibrium magnetization state along the in-plane $x$ direction, the energy barrier is calculated by the energy difference between $x$ and $y / z$ directions when $E=0$, i.e., $\Delta G_{\text {in }}=\frac{1}{2} \mu_{0} M_{\mathrm{s}}^{2} V\left[\min \left(N_{y}, N_{z}-\frac{2 K_{E=0}}{\mu_{0} M_{s}^{2}}\right)-N_{x}\right]$, in which the volume $V=\pi a b t$ and $N_{x}, N_{y}$, and $N_{z}$ is the demagnetization factor along $x$, $y$, and $z$ direction, respectively. Similarly, in the case of initial magnetization along the perpendicular $z$ direction, the energy barrier can be calculated as $\Delta G_{\mathrm{pe}}=\frac{1}{2} \mu_{0} M_{\mathrm{s}}^{2} V\left[N_{x}-\left(N_{z}-\frac{2 K_{E=0}}{\mu_{0} M_{\mathrm{s}}^{2}}\right)\right]$. For simplicity, the elliptical geometry with $a=2 b$ is considered. From these equations, we can determine the region in which $\Delta G$ is higher than $40 k_{\mathrm{B}} T(T=300 \mathrm{~K})$, as shown by the contour plots in Fig. $4(n=11)$ and Fig. S4 (in SI) $(n=5,7,9)$. It can be seen from Fig. 4a that for an in-plane switching with the initial magnetization along $x$ axis, suitable $\varepsilon_{\mathrm{MgO}}$ larger than approximately $-3.313 \%$ and
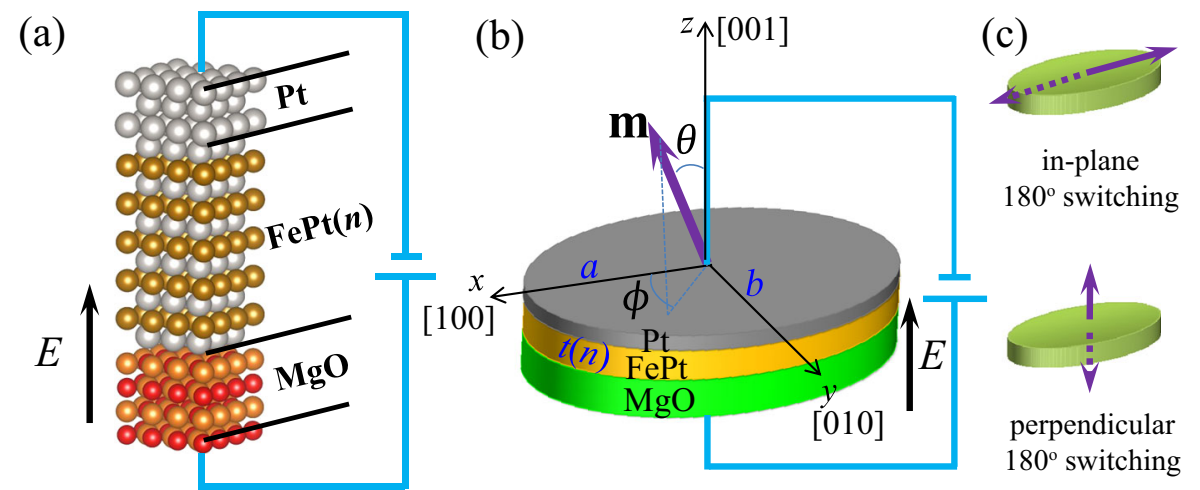

Fig. 1 Material model system for a first-principles calculations and $\mathbf{b}$ magnetization dynamics simulation. $\mathbf{c}$ Illustrations of in-plane and perpendicular $180^{\circ}$ switching 


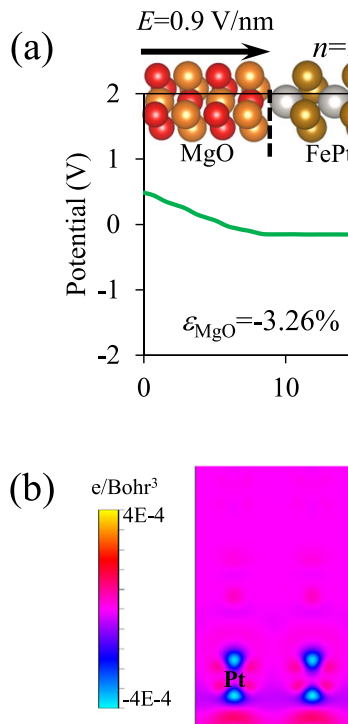

$(020)$
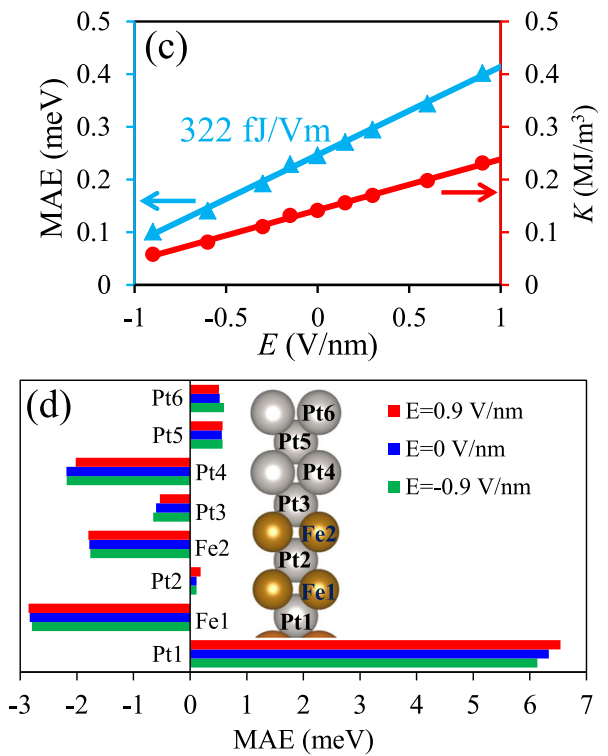

Fig. 2 Typical first-principles results for $n=5$ and $\varepsilon_{\mathrm{MgO}}=-3.26 \%$. a Electrostatic potential distribution. $\mathbf{b}$ Electric field $(E=0.9 \mathrm{~V} / \mathrm{nm})$ induced charge density change near Pt and Fe atoms close to the MgO/FePt interface. c MAE as a function of the external electric field. d Atomresolved MAE
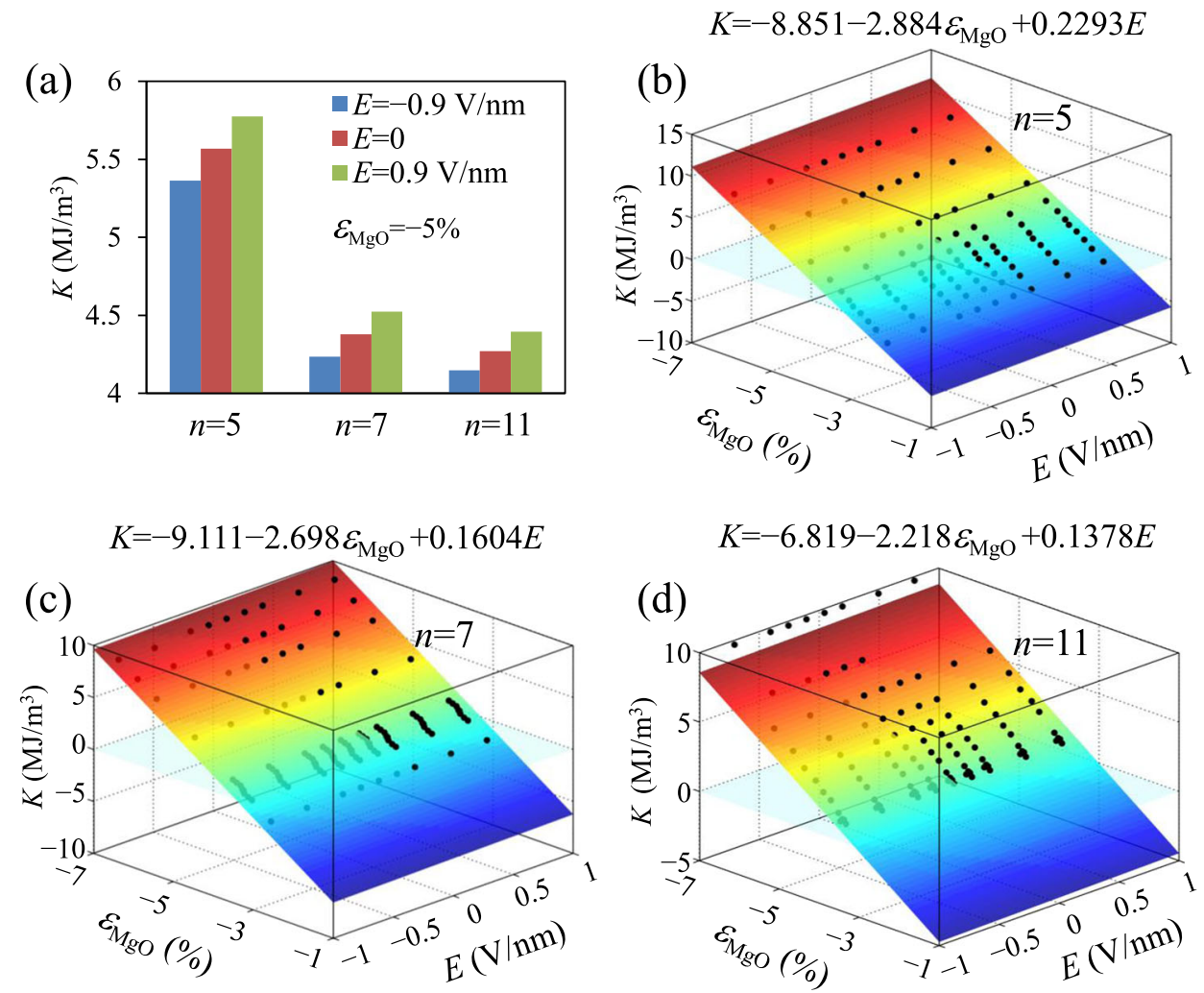

Fig. 3 a $K$ for different layer number $n$ of FePt. $K$ as functions of $\varepsilon_{\mathrm{MgO}}$ and $E$ when $\mathbf{b} n=5$, $\mathbf{c} n=7$, and $\mathbf{d} n=11$. The equations in $\mathbf{b}$-d are obtained by linear fitting

a larger than $\sim 44 \mathrm{~nm}$ are necessary for a energy barrier above $40 k_{\mathrm{B}} T$. The critical value of $a$ for an in-plane switching increases with the decrease of $n$ (Fig. S4 in SI). In contrast, a energy barrier over $40 k_{\mathrm{B}} T$ for a perpendicular switching with the initial magnetization along $z$ axis requires $\varepsilon_{\mathrm{MgO}}$ smaller than approximately $-3.313 \%$, but its dependence on $a$ is much weaker, as shown in Fig. 4b. Based on the results in Fig. 4, the geometry parameter $a=2 b=46 \mathrm{~nm}$ is chosen for the following study of case $n=11$. This geometry makes it possible that the energy barrier of the initial equilibrium magnetization in both in-plane and perpendicular switching cases is beyond $40 k_{\mathrm{B}} T$ for practical applications.

Heretofore we have figured out the strain and geometry conditions for a stable equilibrium magnetization $(E=0)$ with 


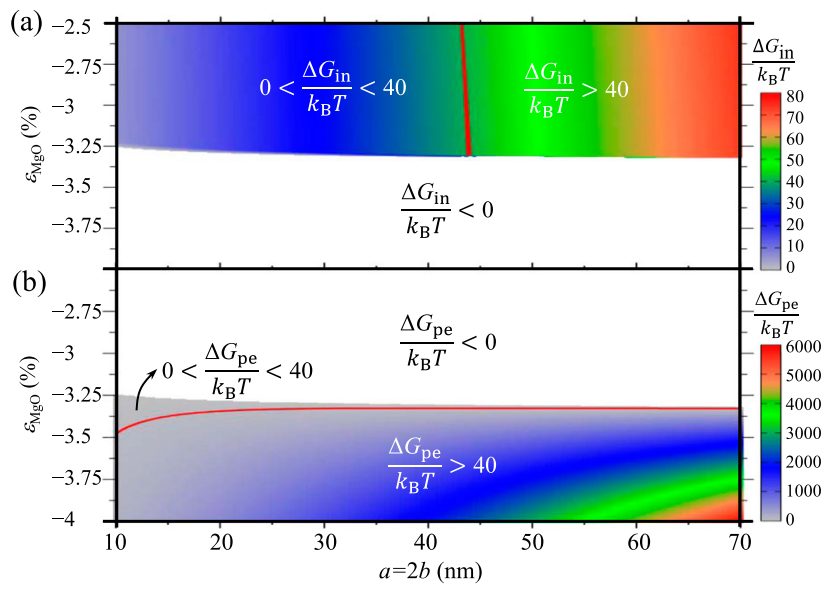

Fig. 4 Energy barrier of the initial equilibrium magnetization $(E=0)$ with $n=11$ : a initial magnetization along the in-plane $x$ direction; $\mathbf{b}$ initial magnetization along the perpendicular $z$ direction. $k_{\mathrm{B}}$ is Boltzmann constant and $T=300 \mathrm{~K}$

energy barrier higher than $40 k_{\mathrm{B}} T$ from the viewpoint of practical applications. However, for the voltage-driven $180^{\circ}$ switching, the E-induced energy change $\left(\left|\Delta G^{\mathrm{k}}\right|\right)$ and magnetization dynamics should be deliberated. Through the total energy analysis (Eq. (1) in Methods), we firstly clarify how large $\varepsilon_{\mathrm{MgO}}$ and $E$ make $\left|\Delta G^{\mathrm{k}}\right|$ beyond $40 k_{\mathrm{B}} T$ and the $180^{\circ}$ switching possible. $E$-induced energy change can be easily calculated as $\left|\Delta G^{\mathrm{k}}\right|=\left(K-K_{E=0}\right) V$. In the case of an in-plane $180^{\circ}$ switching, the magnetic easy axis is along the semimajor axis $x$ when there is no electric field. The in-plane $180^{\circ}$ switching means the magnetization switching from the positive semimajor axis to the negative one, or vice versa. These two equivalent in-plane easy axes guarantee a non-volatile effect, i.e., when the $180^{\circ}$ switching is achieved the associated magnetization state will be kept even after the removal of the electric field. The necessary conditions for the $180^{\circ}$ switching imply two criteria. Firstly, $K$ is small enough to ensure a magnetic easy plane when there is no electric field, i.e., $N_{x}<N_{z}-2 K_{E=0} / \mu_{0} M_{s}^{2}$ (Eq. (1) in Methods section). Secondly, after applying a positive electric field, $K$ is increased to be high enough to achieve a perpendicular easy axis, i.e., $N_{x}>N_{z}-2 K_{E>0} / \mu_{0} M_{s}^{2}$. Therefore, the in-plane switching requires the criterion $N_{z}-2 K_{E=0} / \mu_{0} M_{s}^{2}>N_{x}>N_{z}-2 K_{E>0} / \mu_{0} M_{s}^{2}$. On the contrary, the perpendicular $180^{\circ}$ switching denotes the magnetization switching from the positive perpendicular axis $z$ to the negative one, or vice versa. Two criteria required by the necessary conditions for the perpendicular $180^{\circ}$ switching are opposite to those for the in-plane $180^{\circ}$ switching. On the one hand, without electric field $K$ should be large enough to ensure a perpendicular magnetic easy axis. On the other hand, after a negative electric field is applied, $K$ should be decreased to be small enough to ensure an in-plane easy axis. So the criterion $N_{z}-2 K_{E<0} / \mu_{0} M_{s}^{2}>N_{x}>N_{z}-2 K_{E=0} / \mu_{0} M_{s}^{2}$ should be fulfilled for the perpendicular switching. Combing these two switching criteria, the relation $\left|\Delta G^{\mathrm{k}}\right|>40 k_{\mathrm{B}} T$, and the initial energy barrier $\Delta G_{\text {in }}$ $\left(\Delta G_{\mathrm{pe}}\right)>40 k_{\mathrm{B}} T$ in Fig. 4, we can estimate the necessary conditions for the possible $180^{\circ}$ switching, as shown in Fig. 5 . It can be found that in the triangular regions within a small range of $\varepsilon_{\mathrm{MgO}}$, a positive and negative $E$ potentiates an in-plane and perpendicular $180^{\circ}$ switching, respectively. In a given system with specified layer number and $\varepsilon_{\mathrm{MgO}}$, only one switching mode is possible. There is no overlapped region between in-plane and perpendicular switching modes.

It should be noted that Fig. 5 only presents the necessary rather than the sufficient conditions for a $180^{\circ}$ switching. If the electric field is always kept until the magnetization reaches the

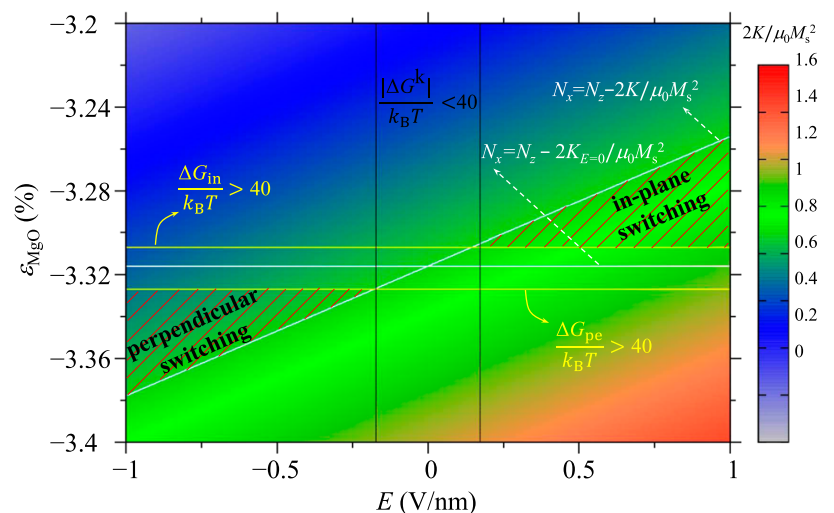

Fig. 5 Necessary conditions for the in-plane and perpendicular $180^{\circ}$ switching $(n=11, a=2 b=46 \mathrm{~nm})$ : switching criteria $N_{z}-2 K_{E=0} / \mu_{0}$ $M_{s}^{2}>N_{x}>N_{z}-2 K_{E>0} / \mu_{0} M_{s}^{2} \quad$ or $\quad N_{z}-2 K_{E<0} / \mu_{0} M_{s}^{2}>N_{x}>N_{z}-2 K_{E=0} /$ $\mu_{0} M_{s}^{2}, E$ induced energy change $\left|\Delta G^{\mathrm{k}}\right|$ beyond $40 k_{\mathrm{B}} T$, initial energy barrier $\Delta G_{\text {in }}\left(\Delta G_{\mathrm{pe}}\right)$ over $40 k_{\mathrm{B}} T$

equilibrium state, only a $90^{\circ}$ switching is achieved. After removing the electric field, in the viewpoint of static analysis the magnetization possesses the equal possibility to revert back or go forwards. Only by using the 3D precessional switching dynamics of magnetization, ${ }^{13-15,18,19,24,25,27}$ a deterministic $180^{\circ}$ switching can be achieved when the pulse width of the electric field is controlled. In the following, we will focus on the switching dynamics in the case of $n=11$ and $\varepsilon_{\mathrm{MgO}}=-3.29 \%$ for an in-plane $180^{\circ}$ switching and $\varepsilon_{\mathrm{MgO}}=-3.34 \%$ for a perpendicular $180^{\circ}$ switching. An electric field pulse with ramp time $\left(t_{r}\right)$ and pulse width $\left(t_{s}\right)$ shown in Fig. 6a is applied to provoke switching. As a first step, we studied the typical switching dynamics at $0 \mathrm{~K}$, as presented in Fig. 6b-e. The spatial and temporal magnetization trajectories in Fig. 6b, d indicate an in-plane and perpendicular $180^{\circ}$ switching, respectively. It can be seen that the precessional dynamics after the removal of $E$ at $t_{s}$ make the magnetization component towards -1 possible. The control of $t_{r}$ and $t_{s}$ is critical for realizing the $180^{\circ}$ switching, as shown in Fig. $6 \mathrm{c}$, e. It can be found that in both the in-plane and perpendicular $180^{\circ}$ switching, a fast switching within $\sim 3 \mathrm{~ns}$ can be attained. The ramp time plays a role in determining whether the $180^{\circ}$ switching fails or succeeds, but has little effects on the switching time which is more influenced by the pulse width. The in-plane $180^{\circ}$ switching allows a much wider range of $E$ pulse width than the perpendicular one. These switching dynamic features are consistent with those from the literature in which the temperature-induced thermal fluctuations are ignored.

However, if the temperature-induced thermal fluctuations are considered, the switching dynamics can be intrinsically altered. Thereby the thermal fluctuation of torque should be considered, as explained in the Methods section. Here we do not perform the temperature-dependent first-principles calculations, but focus on the temperature effect on switching dynamics, i.e., magnetization dynamics including temperature-induced thermal fluctuations. A finite temperature study should be carried out further so that temperature effects can be included in both the parameter prediction by first-principles calculations and the simulation of magnetization dynamics. Even in the equilibrium states without electric field, the magnetization is not exactly aligned along the easy axis. The finite temperature effect makes the magnetization fluctuate within several degrees around the easy axis (Fig. S5 in SI). Figure 7 presents two individual magnetization trajectories at $T=$ $300 \mathrm{~K}$, with other conditions the same as those in Fig. 6b. It is clear that Fig. $6 \mathrm{~b}$ without temperature effect shows a deterministic $180^{\circ}$ 
(a)

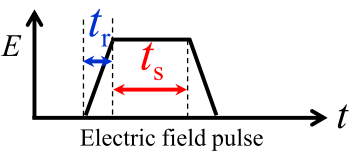

(b)

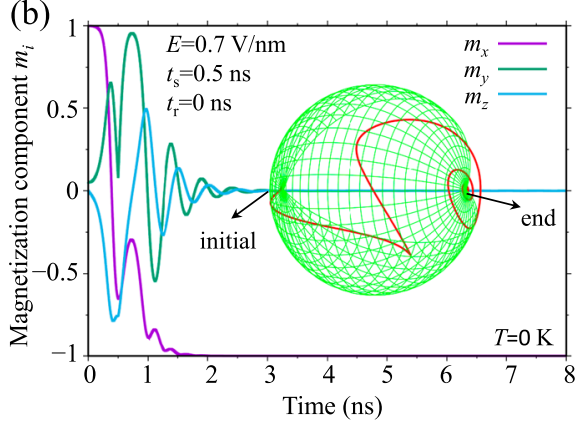

(d)

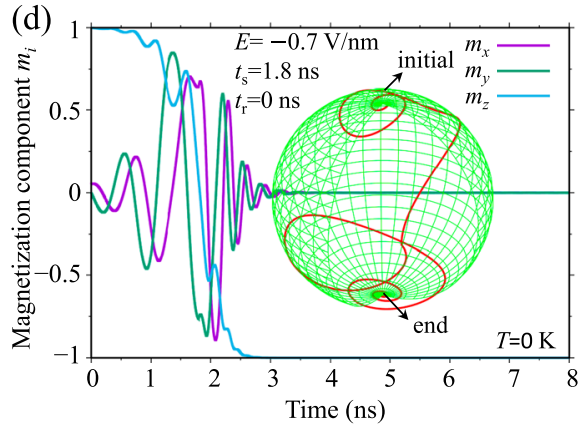

$(\mathrm{c})_{2}$
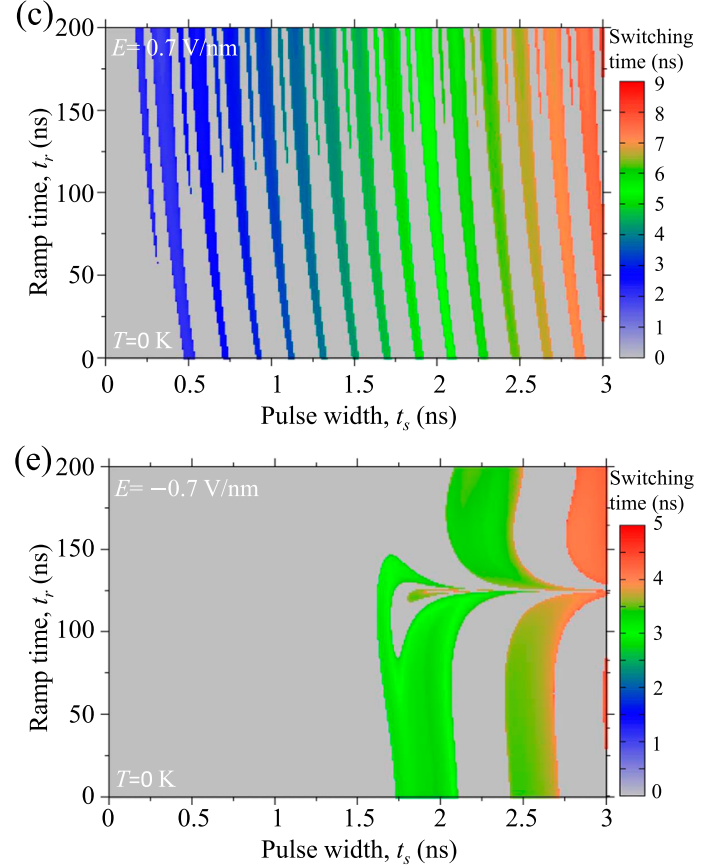

Fig. 6 a Electric field pulse with ramp time $\left(t_{r}\right)$ and pulse width $\left(t_{s}\right)$. Spatial and temporal trajectories of magnetization switching for $\mathbf{b}$ in-plane and $\mathbf{d}$ perpendicular $180^{\circ}$ switching. Switching time as functions of $t_{r}$ and $t_{s}$ for $\mathbf{c}$ in-plane and e perpendicular $180^{\circ}$ switching. The gray region or a switching time of zero in c, e means that the $180^{\circ}$ switching fails. Temperature is set as $0 \mathrm{~K}$
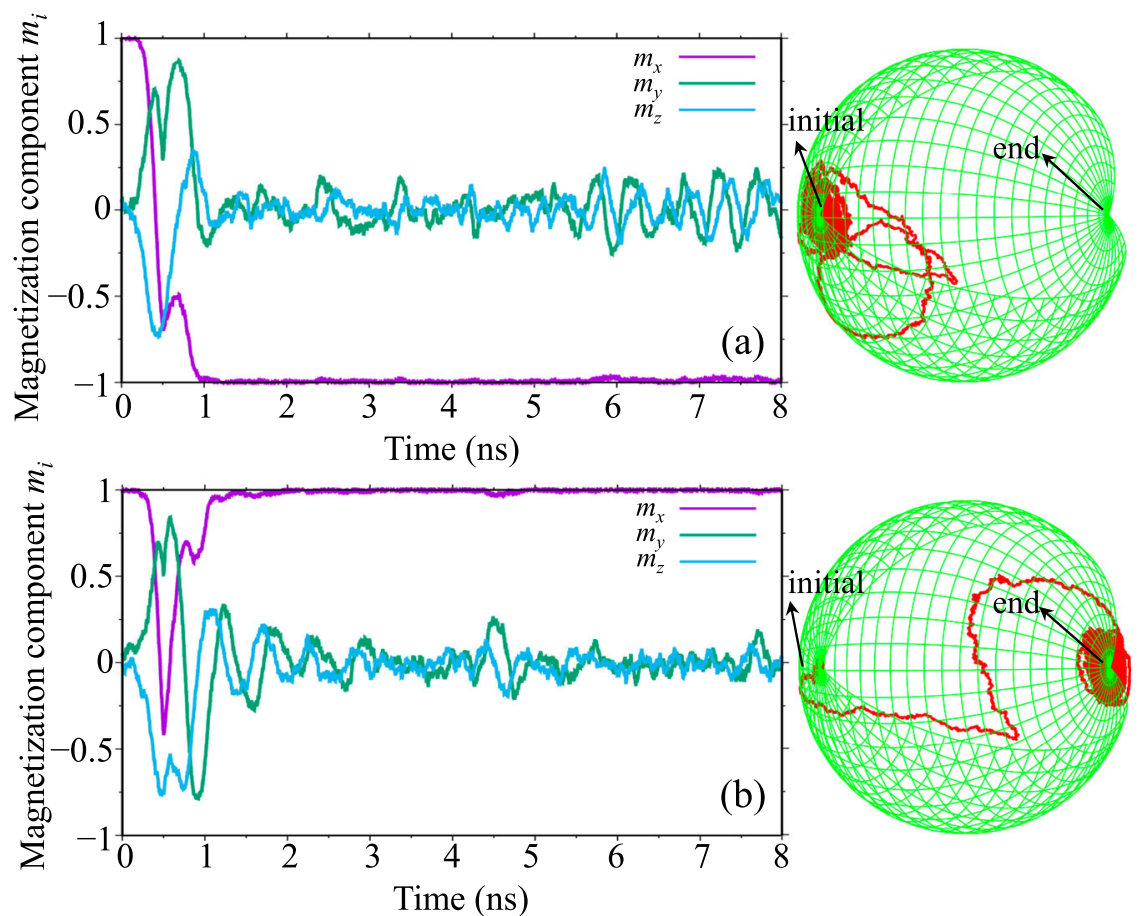

Fig. 7 Two individual trajectories at $T=300 \mathrm{~K}$ for an in-plane $180^{\circ}$ switching $\left(E=0.7 \mathrm{~V} / \mathrm{nm}, t_{\mathrm{s}}=0.5 \mathrm{~ns}, t_{r}=0\right)$ : a $180^{\circ}$ switching succeeds; b $180^{\circ}$ switching fails 
switching. But if $T=300 \mathrm{~K}$ is taken into account, the $180^{\circ}$ switching can either succeed (Fig. 7a) or fail (Fig. 7b). It indicates that the temperature effect disturbs the switching behavior and makes the $180^{\circ}$ switching as probability events. The deterministic switching demonstrated at $0 \mathrm{~K}$ may be not really deterministic at finite temperature, making the previous studies of the $180^{\circ}$ switching at $0 \mathrm{~K}$ to be re-examined.

Figures 8 and 9 compare the switching behaviors at $0 \mathrm{~K}$ with those at $300 \mathrm{~K}$. It can be found from Figs. $8 \mathrm{a}$ and $9 \mathrm{a}$ that at $0 \mathrm{~K}$, the $180^{\circ}$ switching is deterministic and possesses a characteristic switching time. Large electric fields and small pulse width favor a fast switching, with a minimum switching time around $2 \mathrm{~ns}$. When the temperature is involved in the switching dynamics, statistical methodology should be applied to get the $180^{\circ}$ switching probability.
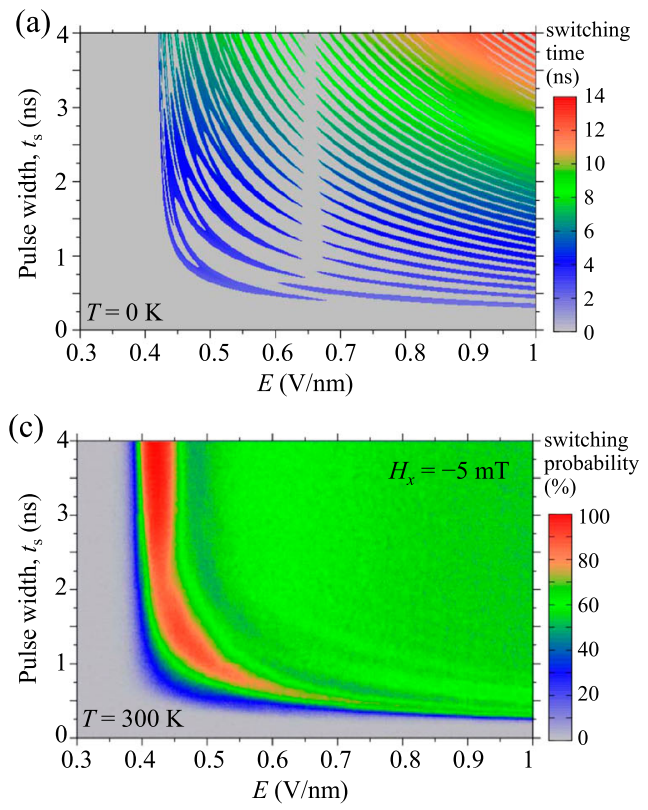

As shown in Figs. 8b, $c$ and $9 b, c$, at $300 \mathrm{~K}$ the switching is not deterministic and a switching probability (the percentage of successful $180^{\circ}$ switching) or error probability (the percentage of unsuccessful $180^{\circ}$ switching) is obtained. For the in-plane $180^{\circ}$ switching, Fig. $8 \mathrm{~b}$ shows that the room temperature makes the $180^{\circ}$ switching with a maximum probability of $70 \%$. If we apply a small bias magnetic field $H_{x}=-5 \mathrm{mT}$ which is the strength of a typical refrigerator magnet, a switching probability above $90 \%$ can be achieved (Fig. 8c). For the perpendicular $180^{\circ}$ switching in Fig. 9, we observe the similar behavior. The magnitude and the pulse width of the electric fields should be carefully designed for a low error probability at room temperature. The region with probability above $\sim 90 \%$ is much wider in Fig. 9c than that in Fig. 8c, indicating more controllability in the perpendicular $180^{\circ}$ switching.
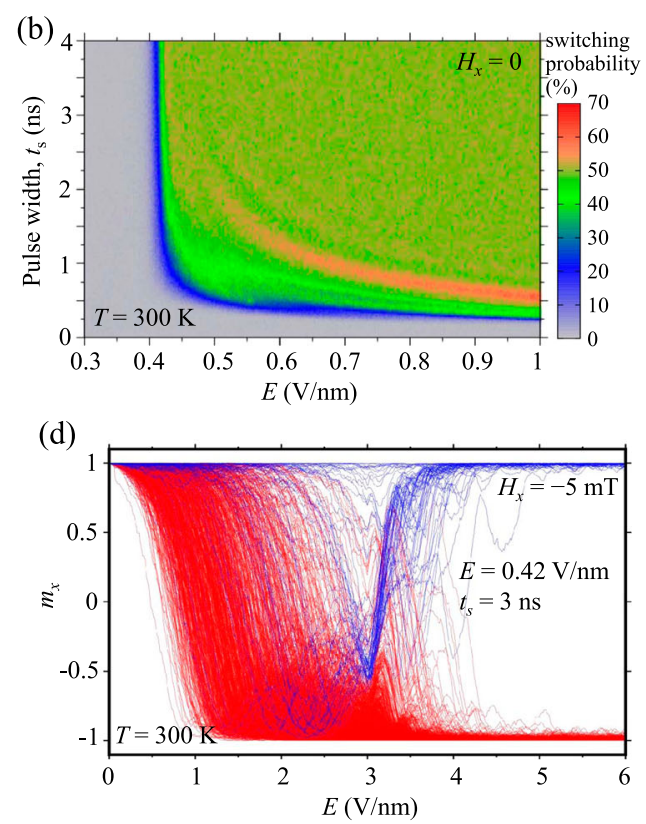

Fig. 8 In-plane $180^{\circ}$ switching. a Switching time at $0 \mathrm{~K}$. Switching probability at $300 \mathrm{~K}$ : b no bias field and $\mathbf{c}$ bias field $H_{x}=-5 \mathrm{mT}$. d 1,000 trajectories with a switching probability of $\sim 93.2 \%$ at $300 \mathrm{~K} . t_{r}=0$
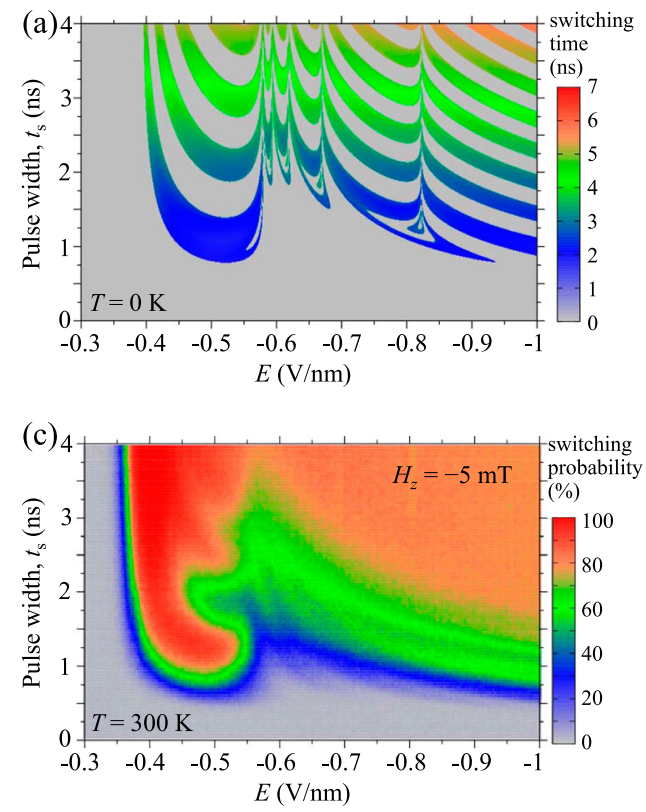
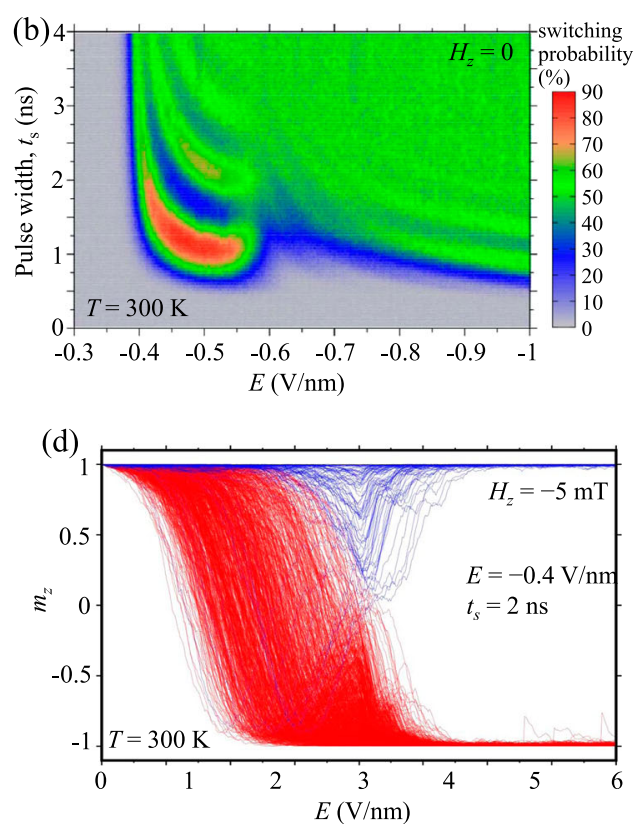

Fig. 9 Perpendicular $180^{\circ}$ switching. a Switching time at $0 \mathrm{~K}$. Switching probability at $300 \mathrm{~K}: \mathbf{b}$ no bias field and $\mathbf{c}$ bias field $H_{z}=-5 \mathrm{mT}$. d 1,000 trajectories with a switching probability of $\sim 91.5 \%$ at $300 \mathrm{~K} . t_{r}=0$ 
For an estimation of switching time at room temperature, as an example we present 1,000 switching trajectories with relatively low error probability for in-plane switching $\left(E=0.42 \mathrm{~V} / \mathrm{nm}\right.$ and $t_{s}=$ $3 \mathrm{~ns}$, Fig. 8d) and perpendicular switching $\left(E=-0.4 \mathrm{~V} / \mathrm{nm}\right.$ and $t_{s}=$ $2 \mathrm{~ns}$, Fig. 9d). The switching time is found to be around $4 \mathrm{~ns}$. The switching probability in Figs. $8 \mathrm{~d}$ and $9 \mathrm{~d}$ is $\sim 93.2 \%$ (error probability $\sim 6.8 \%$ ) and $\sim 91.5 \%$ (error probability $\sim 8.5 \%$ ), respectively. Undeniably, decreasing the error probability as much as possible is desirable. However, the achieved switching probability around $90 \%$ here is still reasonable or may be adequate for memory applications where different on-chip error detection and correction schemes exist.

\section{DISCUSSION}

The voltage-driven charge-mediated perpendicular and in-plane $180^{\circ}$ magnetization switching at $0 \mathrm{~K}$ and room temperature has been studied by using a multiscale theoretical framework which combines first-principles calculations and temperaturedependent magnetization dynamics. For the epitaxial metalmagnet-insulator ( $\mathrm{Pt} / \mathrm{FePt} / \mathrm{MgO})$ hetero-nanostructure as the model system, it is found from first-principles calculations that the interfacial charges induced by electric fields induce a giant modulation of MAE of the nanomagnet. From the temperaturedependent magnetization dynamics using first-principles results, it is found that both in-plane and perpendicular $180^{\circ}$ magnetization switching is possible in the case of suitable epitaxial strain, $E$ pulse width, and $E$ ramp rate. But the temperature effect disturbs the switching behavior and makes the $180^{\circ}$ switching as probability events. The $E$ magnitude and pulse width should be carefully designed for a low-error-probability $180^{\circ}$ switching at room temperature. Statistical analysis indicates that a fast (around $4 \mathrm{~ns}$ ) $180^{\circ}$ switching of low error probability can be achieved at room temperature. This work not only demonstrates a chargemediated way for controlling magnetization by voltage, but also inspires the rational design of miniaturized nanoscale spintronic devices where temperature-induced thermal fluctuation plays a critical role.

\section{METHODS}

The MAE and $M_{s}$ of the system in Fig. 1a are mainly originated from the $L 1_{0}$ ordered FePt layer. The supercell is constructed along the (001) direction, containing $n$-layer FePt on top of four-layer $\mathrm{MgO}$ followed by three-layer $\mathrm{Pt}$ and a 15-Â-thick vacuum layer. The FePt layer number $n$ is chosen to be 5 , 7,9 , and 11 . The electric field is imposed by the dipole layer method, ${ }^{57}$ with the dipole placed in the middle of the vacuum region. The firstprinciples calculations were carried out within the density functional theory and the framework of the projector augmented-wave formalism as implemented in the Vienna ab initio simulation package. ${ }^{58}$ The Perdew-Burke-Ernzerhof exchange-correlation functional in the generalized gradient approximation (GGA) was employed. An energy cutoff of 500 $\mathrm{eV}$ and a Monkhorst-Pack $k$-mesh $31 \times 31 \times 1$, at which a good convergence of MAE was achieved, were utilized. At a certain $\varepsilon_{\mathrm{MgO}}$, the in-plane lattice parameter of the supercell is fixed to be that of the strained $\mathrm{MgO}$ during the relaxation and the atomic positions in the $z$ direction are relaxed. The convergence criteria for the structure relaxation at different $\varepsilon_{\mathrm{MgO}}$ were set as $10^{-6} \mathrm{eV}$ and $2 \mathrm{meV} / \AA$ for the energies and forces, respectively. By using the self-consistent charge density, non-selfconsistent calculations with spin-orbit coupling were performed to get the total energy as a function of the orientation of the quantization axis. $K$ was evaluated as the difference of the total energy per unit FePt volume when the magnetization was along $(100) /(010) \quad(x / y)$ and $(001)(z)$ directions. Positive and negative $K$ indicates perpendicular and in-plane magnetic anisotropy, respectively.

The material system for magnetization dynamics analysis is shown in Fig. 1b. The FePt nanomagnet is an elliptical cylinder with height $t(n)$, semimajor axis $a=46 \mathrm{~nm}$, and semi-minor axis $b=23 \mathrm{~nm}$. For such a small size, a single domain exists and two angles $(\theta, \phi)$ are used to describe the magnetization state. The single-domain state of FePt nanomagnet with magnetic properties from first principles is confirmed by our micromagnetic simulations (Fig. S6 in SI). It should be noted that the electric field induced $K$ change is mainly localized around the interface. This interface effect will be much weaker for the thick film. Here, the FePt nanomagnet is only several atomic layer thick. Since the magnetization behaves coherently (Fig. S6 in $\mathrm{Sl}$ ), we represent all the magnetic moments in these atomic layers by a macro spin with the average saturation magnetization $M_{s}$ and $K$ from first-principles calculations. The similar idea has also been used by previous work. ${ }^{34,41}$ Nevertheless, a more accurate way by atomistic spin simulations which treat each atomic spin separately should be attempted as the next-step work. In this way, the total energy of the FePt elliptical cylinder can be given as the summation of the demagnetization energy and the magnetocrystalline anisotropy energy, i.e.,

$E_{t}=\frac{1}{2} \mu_{0} M_{s}^{2}\left[N_{x} \sin ^{2} \theta \cos ^{2} \phi+N_{y} \sin ^{2} \theta \sin ^{2} \phi+\left(N_{z}-\frac{K}{\frac{1}{2} \mu_{0} M_{s}^{2}}\right) \cos ^{2} \theta\right]$

in which $N_{x}, N_{y}$, and $N_{z}$ is the demagnetization factor along $x, y$, and $z$ direction, respectively, and can be calculated as a function of the geometry size. $^{59}$ Taking the temperature effect as thermal fluctuations, ${ }^{60}$ the temperature-dependent magnetization dynamics is governed by

$$
\begin{gathered}
\dot{\theta}=-\frac{\gamma_{0}}{M_{s}\left(1+\alpha^{2}\right)}\left(a \frac{\partial E_{t}}{\partial \theta}+\frac{1}{\sin \theta} \frac{\partial E_{t}}{\partial \phi}\right) \\
+\frac{1}{2 \tau_{N}} \cot \theta+\frac{1}{\sqrt{\tau_{N} \Delta t}} P_{1} \\
\dot{\phi}=-\frac{\gamma_{0}}{M_{s}\left(1+\alpha^{2}\right) \sin \theta}\left(\frac{a}{\sin \theta} \frac{\partial E_{t}}{\partial \phi}-\frac{\partial E_{t}}{\partial \theta}\right) \\
+\frac{1}{\sin \theta \sqrt{\tau_{N} \Delta t}} P_{2}
\end{gathered}
$$

in which $\gamma_{0}$ is the gyromagnetic ratio constant, $a=0.2$ is the damping coefficient of $L 1_{0} \mathrm{FePt}^{61} \Delta t=0.2 \mathrm{ps}$ is the time step, and $P_{i}(i=1,2)$ is a stochastic process with Gaussian distribution, zero mean value, and completely uncorrelated property in time. The characteristic time $\tau_{N}$ is related to volume $V$ and temperature $T$ as $\tau_{N}^{-1}=2 a \gamma_{0} k_{B} T /\left[M_{s}\left(1+a^{2}\right) V\right]$. The method by using Eqs. (2) and (3) is verified by performing a benchmark test, in which only the thermal fluctuations are considered. In such a simple case, an initial magnetization $\left(m_{x}^{0}, m_{y}^{0}, m_{z}^{0}\right)$ will evolve randomly and the associated average response can be obtained from the theoretical solution of Fokker-Planck equation as $\left\langle m_{i}\right\rangle=m_{i}^{0} \exp \left(-t / \tau_{N}\right)$. Our simulation results are found to agree well with the theoretical solution and the simulation results by MuMax3.62

\section{Data availability}

The authors declare that the data supporting the findings of this study are available within the paper and its Supplementary Information files.

\section{ACKNOWLEDGEMENTS}

The financial supports from the German federal state of Hessen through its excellence programme LOEWE RESPONSE and the German Research Foundation (individual project $\mathrm{Xu} 121 / 7-1$ and the project $\mathrm{Xu} 121 / 4-2$ in the Forscher gruppe FOR1509) are appreciated. The authors greatly acknowledge the access to the Lichtenberg High Performance Computer of Technische Universität Darmstadt, and the support by the German Research Foundation and the Open Access Publishing Fund of Technische Universität Darmstadt.

\section{AUTHOR CONTRIBUTIONS}

M.Y. performed the calculations, analyzed the results, and wrote the manuscript. H. Z. and B.-X.X. analyzed the results and supervised the project. All authors reviewed and approved the manuscript.

\section{ADDITIONAL INFORMATION}

Supplementary information accompanies the paper on the npj Computational Materials website (https://doi.org/10.1038/s41524-017-0043-x).

Competing interests: The authors declare that they have no competing financial interests. 
Publisher's note: Springer Nature remains neutral with regard to jurisdictional claims in published maps and institutional affiliations.

\section{REFERENCES}

1. Hu, J. M., Chen, L. Q. \& Nan, C. W. Multiferroic heterostructures integrating ferroelectric and magnetic materials. Adv. Mater. 28, 15-39 (2016).

2. Tiercelin, N. et al. Nanomagnetic and Spintronic Devices for Energy-Efficient Memory and Computing Ch. 8, 221-257 (John Wiley \& Sons, 2016)

3. Liu, M. \& Sun, N. X. Voltage control of magnetism in multiferroic heterostructures. Phil. Trans. R. Soc. A 372, 20120439 (2014).

4. Hu, J.-M., Nan, T., Sun, N. X. \& Chen, L.-Q. Multiferroic magnetoelectric nanostructures for novel device applications. MRS Bull. 40, 728-735 (2015).

5. Wang, Y., Hu, J., Lin, Y. \& Nan, C.-W. Multiferroic magnetoelectric composite nanostructures. NPG Asia Mater. 2, 61-68 (2010).

6. Velev, J. P., Jaswal, S. S. \& Tsymbal, E. Y. Multi-ferroic and magnetoelectric materials and interfaces. Phil. Trans. R. Soc. A 369, 3069-3097 (2011).

7. Dong, S., Liu, J.-M., Cheong, S.-W. \& Ren, Z. Multiferroic materials and magnetoelectric physics: symmetry, entanglement, excitation, and topology. Adv. Phys. 64, 519-626 (2015).

8. Scott, J. F. Searching for new ferroelectrics and multiferroics: a users point of view. npj Comput. Mater. 1, 15006 (2015).

9. Velev, J. P., Burton, J. D., Zhuravlev, M. Y. \& Tsymbal, E. Y. Predictive modelling of ferroelectric tunnel junctions. npj Comput. Mater. 2, 16009 (2016).

10. Hu, J.-M., Duan, C.-G., Nan, C.-W. \& Chen, L.-Q. Understanding and designing magnetoelectric heterostructures guided by computation: progresses, remaining questions, and perspectives. npj Comput. Mater. 3, 18 (2017).

11. Peng, R. C. et al. Fast 180 degrees magnetization switching in a strain-mediated multiferroic heterostructure driven by a voltage. Sci. Rep. 6, 27561 (2016).

12. Yi, M. \& Xu, B. X. A constraint-free phase field model for ferromagnetic domain evolution. Proc. R. Soc. A 470, 20140517 (2014).

13. Yi, M., Xu, B.-X. \& Shen, Z. Effects of magnetocrystalline anisotropy and magnetization saturation on the mechanically induced switching in nanomagnets. J. Appl. Phys. 117, 103905 (2015).

14. $\mathrm{Hu}, \mathrm{J}$. M. et al. Purely electric-field-driven perpendicular magnetization reversal. Nano. Lett. 15, 616-622 (2015)

15. Yi, M., Xu, B.-X. \& Shen, Z. $180^{\circ}$ magnetization switching in nanocylinders by a mechanical strain. Extreme Mech. Lett. 3, 66-71 (2015).

16. Wang, J. et al. Electric-field modulation of magnetic properties of Fe films directly grown on $\mathrm{BiScO}_{3}-\mathrm{PbTiO}_{3}$ ceramics. J. Appl. Phys. 107, 083901 (2010).

17. Liu, M. et al. Electrically controlled non-volatile switching of magnetism in multiferroic heterostructures via engineered ferroelastic domain states. NPG Asia Mater. 8, e316 (2016).

18. Yi, M., Xu, B.-X. \& Gross, D. Mechanically induced deterministic $180^{\circ}$ switching in nanomagnets. Mech. Mater. 87, 40-49 (2015).

19. Hu, J.-M., Yang, T. N., Chen, L. Q. \& Nan, C. W. Voltage-driven perpendicular magnetic domain switching in multiferroic nanoislands. J. Appl. Phys. 113, 194301 (2013).

20. $\mathrm{Hu}$, J.-M. \& Nan, C. W. Electric-field-induced magnetic easy-axis reorientation in ferromagnetic/ferroelectric layered heterostructures. Phys. Rev. B 80, 224416 (2009).

21. Buzzi, M. et al. Single domain spin manipulation by electric fields in strain coupled artificial multiferroic nanostructures. Phys. Rev. Lett. 111, 027204 (2013).

22. Ghidini, $\mathrm{M}$. et al. Perpendicular local magnetization under voltage control in $\mathrm{Ni}$ films on ferroelectric $\mathrm{BaTiO}_{3}$ substrates. Adv. Mater. 27, 1460-1465 (2015).

23. Ghidini, M. et al. Non-volatile electrically-driven repeatable magnetization reversal with no applied magnetic field. Nat. Commun. 4, 1453 (2013).

24. Roy, K., Bandyopadhyay, S. \& Atulasimha, J. Binary switching in a 'symmetric' potential landscape. Sci. Rep. 3, 3038 (2013).

25. Roy, K., Bandyopadhyay, S. \& Atulasimha, J. Switching dynamics of a magnetostrictive single-domain nanomagnet subjected to stress. Phys. Rev. B 83, 224412 (2011).

26. Tiercelin, N. et al. Room temperature magnetoelectric memory cell using stressmediated magnetoelastic switching in nanostructured multilayers. Appl. Phys. Lett. 99, 192507 (2011).

27. Roy, K., Bandyopadhyay, S. \& Atulasimha, J. Energy dissipation and switching delay in stress-induced switching of multiferroic nanomagnets in the presence of thermal fluctuations. J. Appl. Phys. 112, 023914 (2012).

28. Brintlinger, T. et al. In situ observation of reversible nanomagnetic switching induced by electric fields. Nano. Lett. 10, 1219-1223 (2010).

29. Wang, J. J. et al. Effect of strain on voltage-controlled magnetism in $\mathrm{BiFeO}_{3}$-based heterostructures. Sci. Rep. 4, 4553 (2014).

30. Wang, J. J. et al. Full 180 degrees magnetization reversal with electric fields. Sci. Rep. 4, 7507 (2014).
31. Ong, P. V. et al. Giant voltage modulation of magnetic anisotropy in strained heavy metal/magnet/insulator heterostructures. Phys. Rev. B 92, 020407 (R) (2015).

32. Ong, P. V., Kioussis, N., Amiri, P. K. \& Wang, K. L. Electric-field-driven magnetization switching and nonlinear magnetoelasticity in $\mathrm{Au} / \mathrm{FeCo} / \mathrm{MgO}$ heterostructures. Sci. Rep. 6, 29815 (2016).

33. Weisheit, M. et al. Electric field-induced modification of magnetism in thin-film ferromagnets. Science 315, 349-351 (2007).

34. Zhu, W., Xiao, D., Liu, Y., Gong, S. J. \& Duan, C. G. Picosecond electric field pulse induced coherent magnetic switching in $\mathrm{MgO} / \mathrm{FePt} / \mathrm{Pt}(001)$-based tunnel junctions: a multiscale study. Sci. Rep. 4, 4117 (2014).

35. Rondinelli, J. M., Stengel, M. \& Spaldin, N. A. Carrier-mediated magnetoelectricity in complex oxide heterostructures. Nat. Nanotechnol. 3, 46-50 (2008).

36. Duan, C. G. et al. Surface magnetoelectric effect in ferromagnetic metal films. Phys. Rev. Lett. 101, 137201 (2008).

37. Maruyama, T. et al. Large voltage-induced magnetic anisotropy change in a few atomic layers of iron. Nat. Nanotechnol. 4, 158-161 (2009)

38. Niranjan, M. K., Duan, C.-G., Jaswal, S. S. \& Tsymbal, E. Y. Electric field effect on magnetization at the Fe/MgO(001) interface. Appl. Phys. Lett. 96, 222504 (2010).

39. Chiba, D. et al. Magnetization vector manipulation by electric fields. Nature $\mathbf{4 5 5}$, 515-518 (2008).

40. Wang, W. G., Li, M., Hageman, S. \& Chien, C. L. Electric-field-assisted switching in magnetic tunnel junctions. Nat. Mater. 11, 64-68 (2011).

41. Shiota, Y. et al. Induction of coherent magnetization switching in a few atomic layers of FeCo using voltage pulses. Nat. Mater. 11, 39-43 (2011).

42. Zhou, Z. et al. Interfacial charge-mediated non-volatile magnetoelectric coupling in $\mathrm{Co}_{0.3} \mathrm{Fe}_{0.7} / \mathrm{Ba}_{0.6} \mathrm{Sr}_{0.4} \mathrm{TiO}_{3} / \mathrm{Nb}: \mathrm{SrTiO}_{3}$ multiferroic heterostructures. Sci. Rep. 5, 7740 (2015)

43. Yang, S. W. et al. Non-volatile 180 degrees magnetization reversal by an electric field in multiferroic heterostructures. Adv. Mater. 26, 7091-7095 (2014).

44. Duan, C. G., Jaswal, S. S. \& Tsymbal, E. Y. Predicted magnetoelectric effect in Fe/ $\mathrm{BaTiO}_{3}$ multilayers: ferroelectric control of magnetism. Phys. Rev. Lett. 97, 047201 (2006).

45. Fechner, M. et al. Magnetic phase transition in two-phase multiferroics predicted from first principles. Phys. Rev. B 78, 212406 (2008).

46. Duan, C.-G. et al. Tailoring magnetic anisotropy at the ferromagnetic/ferroelectric interface. Appl. Phys. Lett. 92, 122905 (2008).

47. Yamauchi, K., Sanyal, B. \& Picozzi, S. Interface effects at a half-metal/ferroelectric junction. Appl. Phys. Lett. 91, 062506 (2007).

48. Duan, C. G., Sabirianov, R. F., Mei, W. N., Jaswal, S. S. \& Tsymbal, E. Y. Interface effect on ferroelectricity at the nanoscale. Nano. Lett. 6, 483-487 (2006).

49. Fechner, M., Zahn, P., Ostanin, S., Bibes, M. \& Mertig, I. Switching magnetization by 180 degrees with an electric field. Phys. Rev. Lett. 108, 197206 (2012).

50. Chu, Y. H. et al. Electric-field control of local ferromagnetism using a magnetoelectric multiferroic. Nat. Mater. 7, 478-482 (2008).

51. Heron, J. T. et al. Deterministic switching of ferromagnetism at room temperature using an electric field. Nature 516, 370-373 (2014).

52. Wang, J. J. et al. Magnetization reversal by out-of-plane voltage in $\mathrm{BiFeO}_{3}$-based multiferroic heterostructures. Sci. Rep. 5, 10459 (2015).

53. Skomski, R. \& Coey, J. M. D. Giant energy product in nanostructured two-phase magnets. Phys. Rev. B 48, 15812 (1993).

54. Kim, K. H. et al. Epitaxial growth of $\mathrm{MgO} / \mathrm{TiN}$ multilayers on $\mathrm{Cu}$. Vacuum 83, 897-901 (2009).

55. Jeon, K.-R., Park, C.-Y. \& Shin, S.-C. Epitaxial growth of $\mathrm{MgO}$ and $\mathrm{CoFe} / \mathrm{MgO}$ on $\mathrm{Ge}$ (001) substrates by molecular Beam epitaxy. Cryst. Growth Des. 10, 1346-1350 (2010).

56. Niu, F., Meier, A. L. \& Wessels, B. W. Epitaxial growth and strain relaxation of MgO thin films on Si grown by molecular beam epitaxy. J. Vac. Sci. Technol. B 24, 2586-2591 (2006).

57. Neugebauer, J. \& Scheffler, M. Adsorbate-substrate and adsorbate-adsorbate interactions of $\mathrm{Na}$ and $\mathrm{K}$ adlayers on Al(111). Phys. Rev. B 46, 16067-16080 (1992).

58. Kresse, G. \& Joubert, D. From ultrasoft pseudopotentials to the projector augmented-wave method. Phys. Rev. B 59, 1758-1775 (1999).

59. Beleggia, M., Graef, M. D., Millev, Y. T., Goode, D. A. \& Rowlands, G. Demagnetization factors for elliptic cylinders. J. Phys. D Appl. Phys. 38, 3333-3342 (2005).

60. Brown, W. F. Thermal fluctuations of a single-domain particle. Phys. Rev. 130 , 1677-1686 (1963).

61. Lee, K.-D. et al. Gilbert damping and critical real-space trajectory of $L 1_{0}$-ordered FePt films investigated by magnetic-field-induction and all-optical methods. Appl. Phys. Express 7, 113004 (2014).

62. Vansteenkiste, A. et al. The design and verification of MuMax3. AIP Adv. 4, 107133 (2014). 
Open Access This article is licensed under a Creative Commons Attribution 4.0 International License, which permits use, sharing, adaptation, distribution and reproduction in any medium or format, as long as you give appropriate credit to the original author(s) and the source, provide a link to the Creative Commons license, and indicate if changes were made. The images or other third party material in this article are included in the article's Creative Commons license, unless indicated otherwise in a credit line to the material. If material is not included in the article's Creative Commons license and your intended use is not permitted by statutory regulation or exceeds the permitted use, you will need to obtain permission directly from the copyright holder. To view a copy of this license, visit http://creativecommons. org/licenses/by/4.0/.

(c) The Author(s) 2017 